\title{
Assessment of pattern, morbidities and treatment outcome of admitted neonates in a Regional Hospital SNCU
}

\author{
Authors \\ Ravinder Singh ${ }^{1}$, Shikha Verma ${ }^{2 *}$ \\ ${ }^{1}$ Medical Officer, Dermatology DR RKGMC Hamirpur H.P India \\ ${ }^{2}$ Senior Resident, Department of Pediatrics Dr RPGMC Kangra H.P India \\ *Corresponding Author \\ Shikha Verma
}

\begin{abstract}
Introduction: A country's health is measured in terms of infant mortality. India accounts for 24\% of global neonatal mortality. The neonatal care was revolutionized after the inception of NHM. After reduction in neonatal mortality rate, the goal is to reduce the morbidity of neonates by disease specific intervention. It is important to study the mortality and morbidity pattern as it helps to implement new treatment protocols, interventions, planning and policy making which helps in better survival and improvement in the quality of life among survivors. So, this study aims at identifying the morbidity and mortality pattern in a secondary care SNCU of a Regional Hospital.

Methodology: Hospital data based retrospective study from August 2016 to January 2018.

Results: Total of 868 neonates were analyzed. Male: female ratio was 1.45:1. Low birth weight babies accounted $34 \%$ and pre-term babies around 28\%. Jaundice requiring photo therapy (42.5\%) topped the list of morbidities followed by sepsis (12.9\%) and respiratory distress (5.6\%). Ninety one percent (91\%) neonates were discharged and mortality in this study was $0.46 \%$.

Conclusion: Neonatal jaundice, Sepsis and respiratory distress were the leading causes of morbidity. Neonatal sepsis can be prevented by enforcing strict hand hygiene and aseptic protocols. Low birth weight and prematurity were the significant contributors to morbidity and mortality. Hence antenatal programs to prevent prematurity and low birth weight babies should be strengthened.
\end{abstract}

Keywords: Neonate, Morbidity, Mortality, SNCU.

\section{Introduction}

The Infant Mortality Rate (IMR) is widely accepted as a crude indicator of the overall health scenario of a country or a region. The neonatal period, the first 28 days of life carries the highest risk of mortality per day than any other period during the childhood (NMR).In India the current IMR is 32 (infant deaths per thousand live births) is about one-fourth as compared to IMR which was 129 in 1971. In the last ten years, IMR has witnessed a decline of about $35 \%$ in rural area and $32 \%$ in urban area. Among the States/Union Territories, the IMR ranges from 4 in Nagaland to 48 in Madhya Pradesh for 2018.Seventy percent of total infant deaths and more than half of under-five deaths fall in the neonatal period. Indeed, deaths in the first week alone account for $\sim 45 \%$ of total under-five deaths ${ }^{[1]}$. Of the 25 million babies born in India every year 1 million die, India alone contributes to $25 \%$ of 
neonatal mortality around the world ${ }^{[2]}$. As per the report sheet published in the Lancet, the major direct causes of neonatal mortality are pre-term birth (27\%), infection (26\%), asphyxia(23\%), congenital anomalies (7\%), others (7\%), tetanus $(7 \%)$, and diarrhoea $(3 \%)^{[3]}$. The major causes of neonatal deaths in India include infections, birth asphyxia, and prematurity which contribute to $32.8 \%, 22.3 \%$, and $16.8 \%$ of the total neonatal deaths, respectively ${ }^{[4,5]}$. In India, the neonatal care saw are volution under the auspices of

National Health Mission (NHM) ${ }^{[6]}$. After reduction in NMR, the goal is to reduce the morbidity of neonates by disease specific intervention. So, identifying the pattern of medical illness in an area will help the health care providers to plan service priorities $^{[7]}$. Improvement of the neonatal survival demands the availability and adequacy of trained personnel providing quality neonatal care at SNCUs in secondary and community level hospitals which are accessible to populations at risk in rural areas. As most of the studies on SNCU outcomes in India are from tertiary care centers in cities, there is a need to document the challenges and opportunities that SNCUs face in secondary and community level hospitals. Therefore, this study was conducted with the objective to assess the profile of neonates admitted to a SNCU in a secondary level hospital in Himachal Pradesh.

\section{Methodology}

Study Design and Period: It is a hospital data based retrospective study from August 2016 to January 2018 for a period of 18 months.

Study Place: It was done at SNCU of district Solan, Himachal Pradesh which caters population of district Solan and adjoining areas of district Sirmour and Shimla.

Inclusion Criteria: All babies admitted in our SNCU under 28days of life.

Exclusion Criteria: Inborn neonates not admitted in SNCU.

Statistical Analysis: was done using Microsoft Office Excel@. The standard case definitions of National Neonatology Forum were used.

\section{Results}

A total of 868 neonates formed the study group. Out of them $518(59.67 \%)$ were inborn and $350(40.32 \%)$ were out born admissions. Among these $515(59.33 \%)$ were male and 353(40.66 \%) were female and the male: female sex ratio was 1.45:1. Around sixty five percent 572(65.89\%) babies weighed above $2.5 \mathrm{~kg}$ and low birth weight babies constituted 296(34.10\%). Among this 625(72\%) were term babies and 243(28\%) were preterm. Average duration of hospital stay was 3.8days and maximum number of neonates 569 (65.5\%) stayed in hospital between (1-3days). Analyzing the morbidity pattern, Jaundice requiring phototherapy $210(24.19 \%)$ was the leading cause requiring admission inthe inborn group followed by sepsis $51(5.87 \%)$ and respiratory distress 41 (4.72\%). Similarly among out born admissions, jaundice $159(18.31 \%)$ is the leading cause followed by neonatal sepsis $61(7.02 \%)$. Considering both the groups together, jaundice requiring phototherapy $369(42.51 \%)$ was the commonest morbidity followed by sepsis $112(12.9 \%)$ and respiratory distress 49 (5.64\%). In this study group 790 (91\%) were discharged and $4(0.46 \%)$ died. Out of 868 neonates $63(7.25 \%)$ were referred to higher centre for ventilator support, diagnostic work up and surgical interventions. Around one percent $11(1.26 \%)$ neonates were taken home against medical advice

Table1: Profile of Neonates

\begin{tabular}{|l|c|c|c|}
\hline & Inborn (\%) & Outborn (\%) & Total (\%) \\
\hline No.of babies & $518(59.677)$ & $350(40.322)$ & $868(100)$ \\
\hline Gender & & & \\
\hline Male & $304(35.023)$ & $211(24.308)$ & $515(59.331)$ \\
\hline Female & $214(24.654)$ & $139(16.013)$ & $353(40.668)$ \\
\hline Gestation age & & & \\
\hline$>37$ weeks & $379(43.663)$ & $246(28.341)$ & $625(72.004)$ \\
\hline $34-37$ weeks & $104(11.981)$ & $75(8.640)$ & $179(20.622)$ \\
\hline$<34$ weeks & $35(4.032)$ & $29(3.341)$ & $64(7.373)$ \\
\hline Birth wt. & & & \\
\hline$>2500 g$ & $331(38.133)$ & $241(27.764)$ & $572(66.598)$ \\
\hline $1500-2499 g$ & $174(20.046)$ & $97(11.175)$ & $271(31.221)$ \\
\hline $1000-1499 g$ & $13(1.497)$ & $12(1.382)$ & $25(2.880)$ \\
\hline$<1000 \mathrm{~g}$ & 0 & 0 & 0 \\
\hline Duration of stay & & & \\
\hline$<1$ day & $20(2.304)$ & $15(1.728)$ & $35(4.032)$ \\
\hline $1-3$ days & $348(40.092)$ & $221(25.460)$ & $569(65.552)$ \\
\hline $4-7$ days & $86(9.907)$ & $62(7.142)$ & $148(17.050)$ \\
\hline$>7$ days & $69(7.949)$ & $51(5.875)$ & $120(13.824)$ \\
\hline $\begin{array}{l}\text { Avg. duration of } \\
\text { stay }\end{array}$ & 3.84 & 3.82 & 3.83 \\
\hline
\end{tabular}


Table 2: Morbidity profile of neonates

\begin{tabular}{|l|c|c|c|}
\hline Morbidities & Inborn (\%) & Outborn (\%) & Total (\%) \\
\hline $\begin{array}{l}\text { Respiratory } \\
\text { distress syndrome }\end{array}$ & $8(0.921)$ & $2(0.230)$ & $10(1.152)$ \\
\hline $\begin{array}{l}\text { Meconium } \\
\text { aspiration } \\
\text { syndrome }\end{array}$ & $24(2.764)$ & $2(0.230)$ & $26(2.995)$ \\
\hline $\begin{array}{l}\text { Respiratory other } \\
\text { distress( } \\
\text { causes }\end{array}$ & $41(4.723)$ & $8(0.921)$ & $49(5.645)$ \\
\hline Birth asphyxia & $32(3.686)$ & $5(0.576)$ & $37(4.262)$ \\
\hline Neonatal sepsis & $51(5.875)$ & $61(7.027)$ & $112(12.903)$ \\
\hline $\begin{array}{l}\text { Major congenital } \\
\text { malformations }\end{array}$ & $9(1.036)$ & $1(0.115)$ & $10(1.152)$ \\
\hline $\begin{array}{l}\text { Jaundice requiring } \\
\text { phototherapy }\end{array}$ & $210(24.193)$ & $159(18.317)$ & $369(42.511)$ \\
\hline Hypothermia & $8(0.921)$ & $4(0.460)$ & $12(1.382)$ \\
\hline Hypoglycemia & $20(2.304)$ & 0 & $20(2.304)$ \\
\hline Others & $118(13.594)$ & $105(12.096)$ & $223(25.691$ \\
\hline
\end{tabular}

Table 3: Outcome of Neonates

\begin{tabular}{|l|c|c|c|}
\hline & Inborn & Outborn & Total \\
\hline Discharge & $481(55.414)$ & $309(35.599)$ & $790(91.013)$ \\
\hline Reffered & $36(4.147)$ & $27(3.110)$ & $63(7.258)$ \\
\hline LAMA & $1(0.115)$ & $10(1.152)$ & $11(1.267)$ \\
\hline Died & $3(0.341)$ & $1(0.115)$ & $4(0.460)$ \\
\hline
\end{tabular}

\section{Discussion}

Data pertaining to disease pattern and mortality are useful for health care providers and policy makers to modify and plan treatment or interventions and evaluate the effectiveness of health care initiatives respectively. In our study 518(59.67\%) babies were inborn and $350(40.32 \%)$ were out born which is comparable to other studies ${ }^{[8,9]}$. The number of male neonates 515(59.33\%) admitted in SNCU were more than female neonates $353(40.66 \%)$. The male preponderance in this study is similar to other studies ${ }^{[10-12]}$. This may be because of vulnerability of male neonates and gender preference. In present study out of the total neonates $625(72 \%)$ were term and $243(28 \%)$ were preterm which is comparable to the studies by Rakholia $\mathrm{R}$ et al, and Modi $\mathrm{R}$ et al. ${ }^{[13,14]}$ Most of preterm were of gestation between 34-37 weeks. We observed LBW admission rates $(31.22 \%)$ which is lower than study by Uppal $\mathrm{K}$ et al $(46.87 \%)^{[15]}$. Neonatal jaundice was leading cause of admission in neonates with 210(24.19\%) inborn and $159(18.31 \%)$ of out born neonates. The incidence of jaundice requiring phototherapy in our study is $42.51 \%$.Camilia $\mathrm{R}$ in her review article quoted that prevalence of jaundice ranges between
25 and $50 \%$ of all term newborns with higher percentage in premature babies ${ }^{[16]}$. In our study, sepsis was found very less in both out born babies i.e $61(7 \%)$ and inborn ones51(5.8\%) as compared to study by Malik et al (45.1\%)\% and Shah et al $(41.3 \%)^{[8,17]}$. The variation in occurrence of sepsis depends upon the health practices being followed in the community and competency of health professionals handling new-born during delivery and post-delivery neonatal care. Neonatal jaundice, sepsis and respiratory distress were the top three morbidities found among the neonates in our study. The incidence of birth asphyxia was found to be $37(4.26 \%)$ which is very less as compared to other studies ${ }^{[8,9]}$. The reason for this could be the quality of care by trained SNCU staff in the hospital labour room. The most important finding of this study is the satisfactory recovery and discharge of $91 \%$ neonates which is quite high as compared to other studies $^{[8,13]}$. Standard operating protocols are followed in labour room and SNCU and regularly communicated to the nursing staff. Emergency admissions are managed by medical officer and staff who are available twenty four hours. Essential equipment is well maintained and regularly serviced. We observed $0.46 \%$ mortality in our study. Mortality rates were found higher in some studies probably due to more number of preterm babies and sicker neonates ${ }^{[8,10,13]}$. The mortality rates depend on many factors like obstetric care, location of referral centre, pattern of referral cases, availability of equipments and skilled man power. The proportion of LAMA and the neonates reffered to higher centers was (1.26\%) and (7.25\%) respectively which is comparable to the study by Uppal K et al $(3.03 \%)$ and $(8.46 \%)^{[15]}$. This study has some limitations. The follow up of neonates who went LAMA and those who were referred to other centers was not done. Maternal details were not studied in the present study.

\section{Conclusion}

In our study, neonatal jaundice, sepsis and respiratory distress were the leading causes of morbidity. Most of the morbidities and subsequent 
mortalities can be prevented by developing infrastructure and training staff for providing effective neonatal resuscitation, practice hand hygiene for prevention of sepsis and effective implementation of IMNCI for early diagnosis of danger signs, timely intervention and timely referral to tertiary care centers.

\section{Funding: Nil}

Conflict of interest: None declared.

\section{Reference}

1. Registrar general and census commissioner, India. Sample registration system(SRS) bulletin volume 53 No.1 May,2020.

2. Bryce J, Boschi-Pinto C, Shibuya K, Black RE;WHO Child Health Epidemiology Reference Group. WHO estimates of the causes of death in children. Lancet. 2005 Mar 26-Apr 1; 365 (9465):1147-52.

3. Lawn JE, Cousens S, Zupan J; Lancet Neonatal Survival Steering Team. 4 million neonatal deaths: When? Where? Why? Lancet 2005;365:891-900.

4. ICMR Young Infant Study Group, "Age profile of neonatal deaths," Indian Pediatrics, vol. 45, no. 12, pp. 991-994, 2008.

5. A. T. Bang, V. K. Paul, H. M. Reddy, and S. B. Baitule, "Why do neonates die in rural Gadchiroli, India? (Part I): primary causes of death assigned by neonatologist based on prospectively observed records," Journal of Perinatology, vol.25, supplement 1, pp. S29-S34, 2005.

6. Kumaravel KS, Ganesh J, Balaji J, Pugalendhiraja KV, Ramesh Babu B. A study on impact of NRHM onNeonatal care and clinical profile Neonates admitted ina SNCU of a rural medical college. Journal of evolution of medical and dental sciences.2015; 82(13); 14335.

7. Kalter HD, Hossain M, BurnhamG, Khan NZ, SahaSK et al. Validation of caregiver interviews to diagnose common causes of severe neonatal illness. Indian perinate epidemiol 1999;13; 99-113.

8. Malik S, Gohiya P, Khan IA. Morbidity profile and mortality of neonates admitted in Neonatal Intensive Care Unit of a Central India Teaching Institute: a prospective observational study. J Clin Neonatol. 2016;5(3):168-73.

9. Baruah MN, Panyang PP. Morbidity and mortality profile of newborns admitted to the special care newborn unit (SCNU) of a teaching hospital of upper Assam, India- a three year study. J Med SciClin Res. 2016 Aug;4(08):11689-95.

10. Shakya A, Shrestha D, Shakya H, Shah SC, Dhakal AK. Clinical profile and outcome of neonates admitted to the Neonatal Care Unit at a teaching hospital in Lalitpur, Nepal. Journal of Kathmandu Medical College 2014; 3(4) Issue 10:144-148.

11. Shrestha SP, Shah AK, Prajapati R, YR Sharma YR. Profile of Neonatal Admission At Chitwan Medical College. Journal of Chitwan Medical College 2013; 3(6): 13-16.

12. Jan AZ, Ahmad S, Zahid SB. Clinical Audit of admission pattern and its outcome in a neonatal ICU. Gomal J Med Sci 2013;11:316.

13. Rakholia R, Rawat V, Bano M, Singh G. Neonatal morbidity and mortality of sick newborns admitted in a teaching hospital of Uttarakhand. CHRISMED J Health Res. 2014 Oct 1;1(4):228.

14. Modi R, Modi B, Patel JK, Punitha KM. Study of the Morbidity and the Mortality Pattern in the Neonatal Intensive Care Unit at a Tertiary Care teaching Hospital in Gandhinagar District, Gujarat, India. J Res Med Den Sci. 2015;3(3):208-12.

15. Uppal K, Ashwani N, Jeelani K et.al. Profile of neonatal mortality in SNCU district hospital. Galore International Journal of Health Sciences \& Research. 2019;4(1): 6-8.

16. Camilia R. Martin, Hinkes MT, John P. Cloherty. Neonatal Hyperbilirubinemia. 
Manual of Neonatal Care. 5th ed. Cloherty JP, Stark AR: Lippincott Williams and Wilkins; 2004:185-222.

17. Shah GS, Yadav S, Thapa A, Shah L. Clinical profile and outcome of neonates admitted to neonatal intensive care unit (NICU) at tertiary care centre in Eastern Nepal. J Nepal Paediatr Soc.2013;33(3):17781. 